\title{
Examining the impact of active clearance of chest drainage catheters on postoperative atrial fibrillation
}

\author{
Samuel St-Onge, MD, Walid Ben Ali, MD, PhD, Ismail Bouhout, MD, MSc, Denis Bouchard, MD, PhD,
} Yoan Lamarche, MD, MSc, Louis P. Perrault, MD, PhD, and Philippe Demers, MD, MSc

\begin{abstract}
Objective: Postoperative atrial fibrillation (POAF) is one of the most frequent complications encountered after heart surgery, and significantly increases complications and mortality. An obstructed chest tube, leaving unevacuated blood around the heart and lungs, can lead to atrial inflammation, which can trigger POAF. The aim of this study was to assess the effectiveness of chest drainage incorporating an active tube clearance (ATC) system in reducing the rate of POAF.
\end{abstract}

Methods: This retrospective analysis based on 300 consecutive patients undergoing heart surgery compared 142 patients allocated to an ATC drainage protocol with 158 controls managed with standard chest drainage. Using a 1:1 propensity score match, 214 patients were included in paired analysis (107 in each group). The primary endpoint was POAF.

Results: Unmatched patients managed with ATC chest drainage protocol had a reduction of $34 \%$ in their POAF rate compared with those managed with standard drains $(23 \%$ vs $35 \%, P=.01)$. In the matched cohort, ATC was associated with a reduction of $31 \%$ in the rate of POAF $(24 \%$ vs $35 \%, P=.09)$ and a trend toward shorter postoperative length of stay $(5.0[4.0 ; 7.0]$ vs $6.0[5.0 ; 8.0], P=.08)$. In multivariable analysis, chest drainage with ATC showed a protective effect on POAF with odds ratio of 0.5 (95\% confidence interval, $0.1-0.9 ; P=.02$ ).

Conclusions: The use of an ATC chest drainage protocol may be associated with reduced POAF. Our results suggest that efforts to maintain chest tube patency could be useful to reduce the incidence of POAF. (J Thorac Cardiovasc Surg $2017 ; 154: 501-8)$

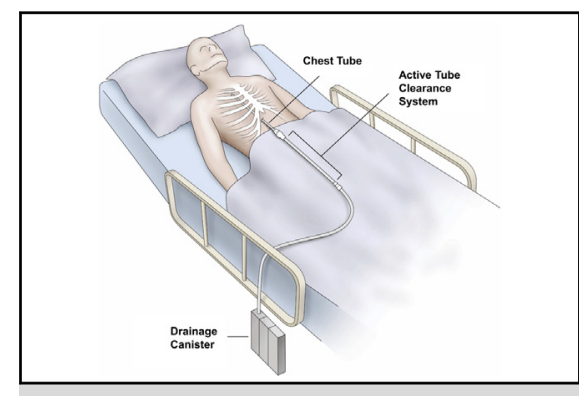

A chest tube clearance device that could potentially prevent postoperative atrial fibrillation.

\section{Central Message}

Active chest tube clearance may be associated with reduction of postoperative atrial fibrillation after heart surgery.

\section{Perspective}

Chest tube clogging is a common and potentially harmful issue after cardiac surgery. We evaluated a drainage protocol using preventive active clearance aimed at alleviating catheter obstruction and demonstrated potential association with reduced postoperative atrial fibrillation. Our findings highlight the relevance of maintaining chest tube patency to improve outcomes after heart surgery.

See Editorial Commentary page 509
Postoperative atrial fibrillation (POAF) is the most common complication after cardiac surgery, with an incidence ranging from $10 \%$ to $65 \%$ depending on the procedure, patient characteristics, definition, and monitoring. ${ }^{1}$ It is associated with hemodynamic compromise, stroke, increased

\footnotetext{
From the Department of Cardiac Surgery, Montreal Heart Institute, Université de Montréal School of Medicine, Montreal, Quebec, Canada.

Received for publication May 19, 2016; revisions received March 1, 2017; accepted for publication March 14, 2017.

Address for reprints: Philippe Demers, MD, MSc, Department of Cardiac Surgery, Montreal Heart Institute, Université de Montréal School of Medicine, 5000 Belanger St, Montreal H1T1C8, Quebec, Canada (E-mail: Philippe.demers@ icm-mhi.org). $0022-5223 / \$ 36.00$

Copyright (C) 2017 Published by Elsevier Inc. on behalf of The American Association for Thoracic Surgery

http://dx.doi.org/10.1016/j.jtcvs.2017.03.046
}

mortality, prolonged hospital stay, and higher health care expenditure. ${ }^{2,3}$ Given the high incidence and important consequences of POAF in this population, prevention strategies have long been sought. Multiple studies tried to demonstrate a reduction in POAF by using pharmacological prophylaxis directed toward modifying neurohormonal regulation of the heart or tapering systemic inflammation and oxidative stress. ${ }^{4-9}$ However, most of these measures have failed to provide sufficient

Scanning this QR code will take you to a supplemental video for the article.

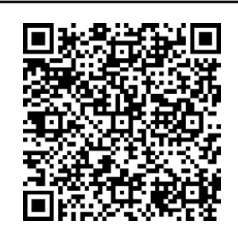




$$
\begin{aligned}
& \text { Abbreviations and Acronyms } \\
& \begin{aligned}
\text { AF } & =\text { atrial fibrillation } \\
\text { ATC } & =\text { active tube clearance } \\
\text { CABG } & =\text { coronary bypass graft } \\
\text { CI } & =\text { confidence interval } \\
\text { COPD } & =\text { chronic obstructive pulmonary disease } \\
\text { ICU } & =\text { intensive care unit } \\
\text { IQR } & =\text { interquartile ranges } \\
\text { LVEF } & =\text { left ventricular ejection fraction } \\
\text { OPCAB } & =\text { off-pump coronary artery bypass } \\
\text { OR } & =\text { odds ratio } \\
\text { POAF } & =\text { postoperative atrial fibrillation } \\
\text { ROS } & =\text { reactive oxygen species }
\end{aligned}
\end{aligned}
$$

effectiveness or are limited in daily clinical practice by their adverse effects, hence the need for a practical, efficient, and widely applicable prevention strategy for POAF.

There is growing evidence that the generation of a proinflammatory and pro-oxidant pericardial milieu driven by blood pooling around the heart is favorable to the generation of arrhythmias in susceptible individuals. ${ }^{10-13}$ In meta-analyses, posterior pericardiotomy proved to be an effective method to prevent POAF by simply shunting blood from the pericardium to the pleura. ${ }^{3,14,15}$ The addition of chest tubes for posterior pericardial drainage was found to provide similar benefits; thus, an alternative to this surgical method could be provided by enhancing chest tube patency, as an association also has been drawn between clogged drainage catheters and higher POAF incidence. ${ }^{15,16}$ Furthermore, a recent propensity-matched investigation demonstrated that the implementation of a universal chest drainage protocol using active tube clearance (ATC), a technology intended to mechanically break up clots in the chest tube lumen and prevent clogging at the bedside in the intensive care unit (ICU), led to a $33 \%$ reduction in the rate of POAF. ${ }^{17}$ To provide further proof confirming that POAF could be prevented by improving actual chest tube management and to emphasize local pericardial inflammation as a new target for prevention, the present study aimed to evaluate the specific effect of a drainage protocol using ATC on POAF incidence in patients undergoing cardiac surgery.

\section{MATERIAL AND METHODS}

\section{Study Population}

The study was undertaken at Montreal Heart Institute. From June 16 to August 11, 2014, 160 consecutive patients were allocated to an ATC chest drainage protocol as part of a special project to evaluate the clinical impact of this technology. After reverting to standard drainage techniques, an unselected cohort of 200 patients meant for comparison was identified from August 12 to September 25, 2014. In total, 60 patients were excluded, $18(11 \%)$ in the
ATC cohort and $42(21 \%)$ in the standard drainage cohort, according to exclusion criteria presented in Figure 1. Data from 300 patients admitted for cardiac surgery in 2014 were analyzed. A total of 142 patients allocated to an ATC drainage protocol were compared with 158 patients managed with standard chest drainage. Moreover, to mitigate the effects of measurable cofounders, patients were matched into 107 pairs according to propensity score matching based on baseline and operative characteristics.

\section{Study Design}

This was a retrospective study of patient data collected in medical records. The primary endpoint was defined as the incidence of an episode of POAF lasting at least 60 minutes or recurrent episodes refractory to standard treatment, on telemetry or electrocardiogram, at any time between post index surgeries through hospital discharge. ${ }^{18}$ Secondary endpoints included mortality, cardiac arrest, permanent stroke, total chest tube output (in milliliters), transfusion requirement, postoperative hospital length of stay, and invasive procedure for intrathoracic fluid removal, a composite outcome including reexploration for bleeding or tamponade, interventions for hemothorax, and drainage procedure for pericardial or pleural effusion, during a time frame ranging from discharge from the operating room to 30 days after index surgery. ${ }^{10,17,19}$ This composite outcome was a way to retrospectively compare the rate of complications that are believed to be related to retained intrathoracic blood and severe enough to require invasive treatment. Our institutional ethics committee approved the use of de-identified data for this study, and a waiver of consent was obtained.

\section{Chest Drainage Technique}

The PleuraFlow Active Tube Clearance Technology System (ClearFlow, Inc, Anaheim, Calif) is a device designed to maximize the permeability of drainage catheters by mechanically breaking up clots or fibrinous debris. It consists of 2 parts: a silicone chest tube positioned in the typical manner and a guide tube inserted between the chest tube and the extension duct to the drainage canister (Figure 2). ${ }^{20}$ Within the guide tube travels an internal guidewire ending with a small loop set at a $105^{\circ}$ angle. This guidewire is magnetically coupled with the external shuttle. By advancing and retracting the external shuttle, the inner wire is moved back and forth inside the lumen, allowing the distal loop to break up clots and enabling nurses to periodically clear the chest tube lumen without compromising sterility. ${ }^{21}$

Patients undergoing cardiac surgery through median sternotomy had 1 or more mediastinal drains positioned anterior to the heart. In the ATC group, one of these drains was a PleuraFlow sized $28 \mathrm{~F}$. Additional pleural tubes were allowed, according to surgeon's preference. In the conventional drainage group, patients had at least 1 standard mediastinal drain sized $28 \mathrm{~F}$ or $32 \mathrm{~F}$ positioned anterior to the heart. Additional Blake drains (Ethicon, Inc, Somerville, NJ) were used, at the discretion of the surgeon, in patients undergoing valvular or aortic procedures. In both groups, when the approach was minimally invasive $(\mathrm{n}=31)$, either ministernotomy or right minithoracotomy, a Blake drain was used combined with a PleuraFlow or a standard chest tube.

The ICU nurses were accustomed and trained to use the ATC system since a pilot study was held at our institution in January $2011 .^{20}$ They were instructed to use the device every 15 minutes during the first 8 hours, the most critical period for postoperative hemorrhage, every 30 minutes for the next 16 hours, and finally every hour until removal of the drain. Because current methods used to clear visible clots from standard chest tubes, such as milking, stripping, and tapping, lack clinical or physiological advantages and are associated with risks of infection and tissue damage, the nurses were instructed not to use these manipulations. ${ }^{22}$ However, if a severe internal occlusion was suspected in standard catheters, direct aspiration was performed by a physician when deemed necessary. 




FIGURE 1. Population flow chart. VAD, Ventricular assist device.

\section{Atrial Fibrillation Management}

Patients were all monitored by continuous telemetry from index surgery through hospital discharge as part of the usual practice. Our local prophylaxis protocol was designed in collaboration with the electrophysiology department and advocated the use of $\beta$-blockade when there was no contraindication, as it constitutes the dominant pharmacological prophylaxis established by both the Canadian Cardiovascular Society and the American Heart Association. ${ }^{23,24}$ Amiodarone, although recommended as a second-line prophylactic agent, was not part of our protocol to avoid adverse effects. Management therapy for an episode of atrial fibrillation (AF) involved amiodarone intravenous, eventually converted to oral administration, and further addition of $\beta$-blocker when not contraindicated.

\section{Statistical Analysis}

A logistic regression predicting the choice of drainage protocol (ATC vs standard) was conducted for all baseline and operative characteristics presented in Table 1 and Table 2 . Seven variables significantly different between ATC and standard drainage in the unmatched population were identified $(P<.10)$. Accordingly, a propensity score was computed to match the ATC group with the standard group (1:1) according to sex, left

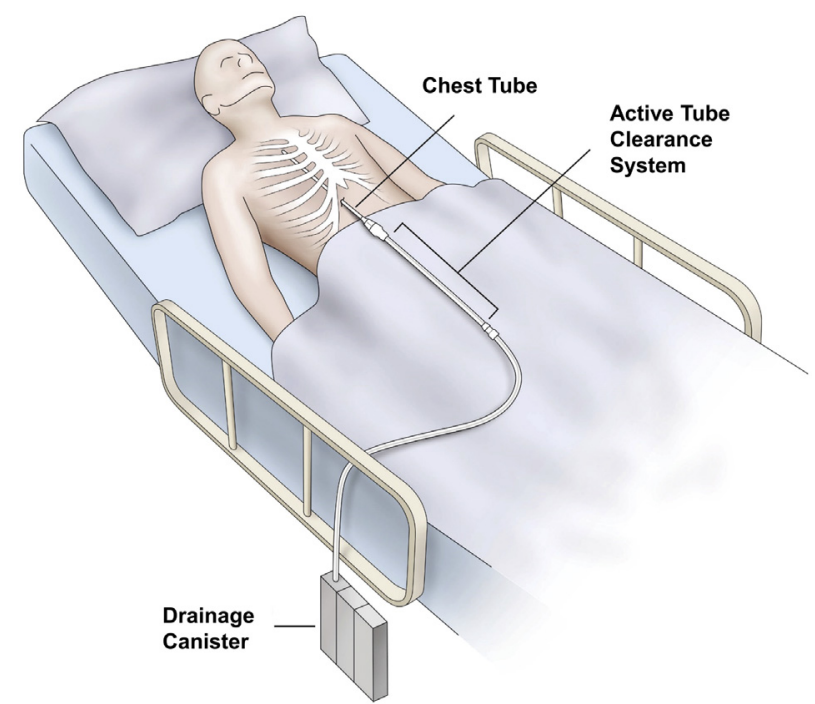

FIGURE 2. Active Tube Clearance System (ClearFlow, Inc, Anaheim, Calif) positioning. ventricular ejection fraction (LVEF) $<30 \%$, preoperative use of statin and $\beta$-blocker, discontinued antiplatelet therapy, off-pump coronary artery bypass (OPCAB), and aortic crossclamp time $>60$ minutes. Patients were matched by using the nearest neighbor method without replacement and a caliper width of 0.25 . The $\mathrm{C}$-statistic for the propensity score model was 0.730 and the Hosmer-Lemeshow test for goodness of fit was 0.827 . The propensity scores kernel density estimation is shown in Figure 3.

As a second step, to include the greater amount of data in the analysis, a logistic regression was used in the general population to estimate the risk of POAF according to the patient's group. Both demographic and operative characteristics were screened as potential confounders. All variables with a $P<.20$ on univariate analysis were considered as having a confounding effect. Variables deemed to be of clinical significance were also considered as potential confounders and were forced in the multivariable model. Moreover, the propensity score was included in the multivariable model as a confounder. A nonautomated variable selection was performed.

Continuous variables were compared between groups using independent $t$ test and presented as mean \pm standard deviation unless normality was ruled out using the Kolmogorov-Smirnov test: in those cases, MannWhitney $U$ test was used and data were expressed as median and interquartile ranges (IQRs). Discrete variables were characterized by frequency with percent and compared using $\chi^{2}$ or Fisher exact test when appropriate. Within the matched pairs, comparison of continuous variables used paired $t$ tests for symmetrically distributed variables and Mann-Whitney $U$ test for skewed variables as categorical variables were compared using McNemar test. A 2-tailed $P<.05$ was considered statistically significant. All analyses were performed with SPSS v. 23.0 (IBM Corp, Armonk, NY).

\section{RESULTS}

\section{Baseline and Operative Characteristics}

Unmatched cohort. Main demographic characteristics are presented in Table 1 as operative characteristics are shown in Table 2. At baseline, there were significant differences with regard to the proportion of men within groups $(78 \%$ vs $63 \%, P=.01)$, preoperative $\beta$-blocker therapy $(56 \%$ vs $68 \%, P=.04$ ), and antiplatelet therapy discontinuation ( $46 \%$ vs $32 \%, P=.01$ ) when comparing the ATC and standard drainage group, respectively. LVEF (55.0 [45.0; 60.0] $\%$ vs $60.0[55.0 ; 60.0] \%, P=.07)$ and preoperative use of statin $(68 \%$ vs $78 \%, P=.06)$ differed between groups, although not statistically significantly. 
TABLE 1. Baseline characteristics

\begin{tabular}{|c|c|c|c|c|c|c|}
\hline \multirow[b]{2}{*}{ Variable } & \multicolumn{3}{|c|}{ Unadjusted data } & \multicolumn{3}{|c|}{ Propensity score matched data } \\
\hline & $\operatorname{ATC}(n=142)$ & Standard $(n=158)$ & $\boldsymbol{P}$ & $\operatorname{ATC}(\mathbf{n}=107)$ & Standard $(n=107)$ & $P$ \\
\hline Age, y & $66.1 \pm 11.0$ & $65.7 \pm 12.1$ & .79 & $66.8 \pm 11.1$ & $65.1 \pm 12.6$ & .39 \\
\hline Male & $110(78)$ & $100(63)$ & .01 & $76(71)$ & $78(73)$ & .76 \\
\hline Body mass index, $\mathrm{kg} / \mathrm{m}^{2}$ & $29.8 \pm 5.1$ & $28.7 \pm 6.0$ & .10 & $28.3 \pm 5.1$ & $29.3 \pm 5.3$ & .15 \\
\hline LVEF & $55.0(45.0 ; 60.0)$ & $60.0(55.0 ; 60.0)$ & .07 & $55.0(50.0 ; 60.0)$ & $56.0(50.0 ; 60.0)$ & .50 \\
\hline Arterial hypertension & $120(85)$ & $132(84)$ & .82 & $90(84)$ & $93(87)$ & .56 \\
\hline Diabetes mellitus & $48(34)$ & $64(41)$ & .23 & $33(31)$ & $43(40)$ & .15 \\
\hline Coronary artery disease & $107(75)$ & $128(81)$ & .24 & $80(75)$ & $87(81)$ & .25 \\
\hline Peripheral arterial disease & $19(13)$ & $20(13)$ & .85 & $13(12)$ & $9(8)$ & .37 \\
\hline Liver dysfunction & $4(3)$ & $3(2)$ & .71 & $2(2)$ & $1(1)$ & $>.99$ \\
\hline COPD & $26(18)$ & $18(11)$ & .09 & $18(17)$ & $14(13)$ & .44 \\
\hline Dyslipidemia & $114(80)$ & $127(80)$ & .98 & $90(84)$ & $85(79)$ & .38 \\
\hline NYHA $\geq$ III & $10(7)$ & $9(6)$ & .63 & $9(8)$ & $7(7)$ & .60 \\
\hline Unstable angina & $51(36)$ & $61(39)$ & .63 & $37(35)$ & $45(42)$ & .26 \\
\hline Recent MI & $30(21)$ & $36(23)$ & .73 & $19(18)$ & $24(22)$ & .39 \\
\hline Chronic kidney disease stage $\geq 3$ & $22(16)$ & $23(15)$ & .82 & $14(13)$ & $16(15)$ & .69 \\
\hline Dialysis & $1(<1)$ & $2(1)$ & $>.99$ & $1(1)$ & $1(1)$ & $>.99$ \\
\hline \multicolumn{7}{|l|}{ Medication } \\
\hline Statin & $97(68)$ & $121(78)$ & .06 & $80(75)$ & $83(78)$ & .63 \\
\hline$\beta$-blocker & $80(56)$ & $105(68)$ & .04 & $66(62)$ & $67(63)$ & .89 \\
\hline $\mathrm{CCB}$ & $36(25)$ & $49(32)$ & .23 & $28(26)$ & $27(26)$ & .94 \\
\hline Amiodarone & $2(1)$ & $0(0)$ & .23 & $1(1)$ & $0(0)$ & $>.99$ \\
\hline Aspirin & $106(75)$ & $121(77)$ & .70 & $80(75)$ & $79(74)$ & .88 \\
\hline Warfarin & $1(<1)$ & $4(3)$ & .37 & $1(1)$ & $2(2)$ & $>.99$ \\
\hline Heparin & $68(48)$ & $63(40)$ & .16 & $43(40)$ & $40(37)$ & .67 \\
\hline Discontinued antiplatelet therapy & $65(46)$ & $50(32)$ & .01 & $43(40)$ & $36(34)$ & .32 \\
\hline Discontinued heparin & $19(13)$ & $21(13)$ & .98 & $15(14)$ & $13(12)$ & .69 \\
\hline
\end{tabular}

Categorical variables are presented as frequency (\%); continuous variables are presented as mean \pm SD when they were normally distributed or as median (IQR) otherwise. $A T C$, Active tube clearance; $L V E F$, left ventricular ejection fraction; COPD, chronic obstructive pulmonary disease; NYHA, New York Heart Association; $M I$, myocardial infarction; $C C B$, calcium channel blocker.

More patients underwent $\mathrm{OPCAB}$ in the standard drainage group ( $4 \%$ vs $17 \%, P=.01)$. Aortic crossclamping times were significantly longer in the standard drainage group (46.0 [29.0; 65.0] minutes vs 54.5 [37.0; 73.0] minutes, $P=.03$ ).
Matched cohort. The propensity score matching resulted in a total cohort of 214 patients allocated to ATC chest tube management or standard drainage protocol. Both groups of 107 patients were well balanced in terms of baseline (Table 1) and operative characteristics (Table 2).

TABLE 2. Operative characteristics

\begin{tabular}{|c|c|c|c|c|c|c|}
\hline \multirow[b]{2}{*}{ Variable } & \multicolumn{3}{|c|}{ Unadjusted data } & \multicolumn{3}{|c|}{ Propensity score matched data } \\
\hline & $\operatorname{ATC}(n=142)$ & Standard $(n=158)$ & $\boldsymbol{P}$ & $\operatorname{ATC}(n=107)$ & Standard $(n=107)$ & $P$ \\
\hline Elective status & $127(89)$ & $138(87)$ & .57 & $100(94)$ & $98(92)$ & .60 \\
\hline Previous cardiac surgery & $7(5)$ & $9(6)$ & .77 & $6(6)$ & $4(4)$ & .52 \\
\hline OPCAB & $6(4)$ & $26(17)$ & .01 & $6(6)$ & $4(4)$ & .52 \\
\hline $\mathrm{CPB}$ time, $\min$ & $69.0(51.0 ; 88.5)$ & $74.0(56.0 ; 92.5)$ & .21 & $74.5(52.0 ; 96.3)$ & $69.0(52.0 ; 92.0)$ & .60 \\
\hline Aortic crossclamp time, min & $46.0(29.0: 65.0)$ & $54.5(37.0 ; 73.0)$ & .03 & $51.0(32.0 ; 69.0)$ & $48.0(30.0 ; 72.0)$ & .94 \\
\hline Valve replacement & $45(32)$ & $55(35)$ & .57 & $40(37)$ & $40(37)$ & $>.99$ \\
\hline Aortic valve & $38(27)$ & $44(28)$ & .83 & $33(31)$ & $31(29)$ & .77 \\
\hline Mitral valve & $8(6)$ & $14(9)$ & .28 & $8(8)$ & $11(10)$ & .47 \\
\hline CABG alone & $89(63)$ & $93(59)$ & .50 & $64(60)$ & $61(57)$ & .68 \\
\hline CABG + Valve replacement & $17(12)$ & $22(14)$ & .62 & $16(15)$ & $18(17)$ & .71 \\
\hline Ascending aortic procedure & $6(4)$ & $7(4)$ & .93 & $5(5)$ & $4(4)$ & $>.99$ \\
\hline Minimally invasive approach & $11(8)$ & $14(9)$ & .73 & $10(9)$ & $9(8)$ & .81 \\
\hline Ministernotomy & $7(5)$ & $7(4)$ & .84 & $6(6)$ & $4(4)$ & .52 \\
\hline Right minithoracotomy & $4(3)$ & $7(4)$ & .46 & $4(4)$ & $5(5)$ & $>.99$ \\
\hline
\end{tabular}

Categorical variables are presented as frequency (\%); continuous variables are presented as median (IQR). ATC, Active tube clearance; OPCAB, off-pump coronary bypass; $C P B$, cardiopulmonary bypass; $C A B G$, coronary artery bypass graft. 


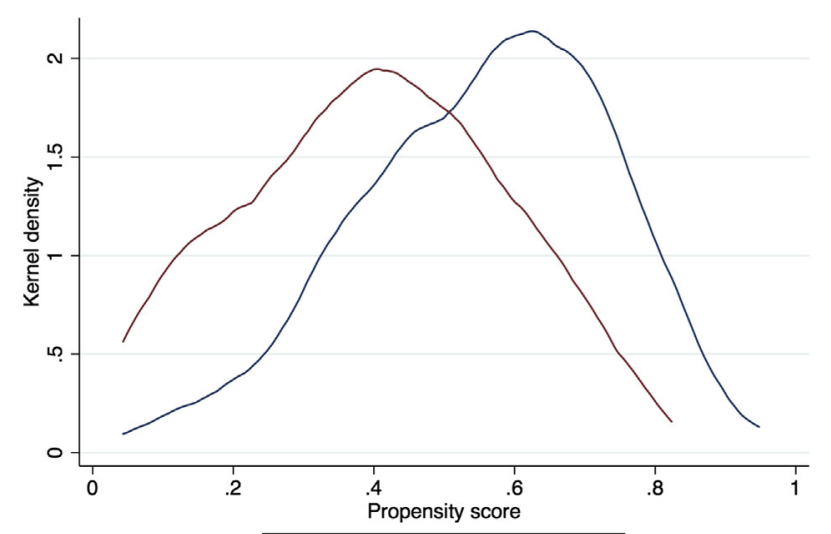

A

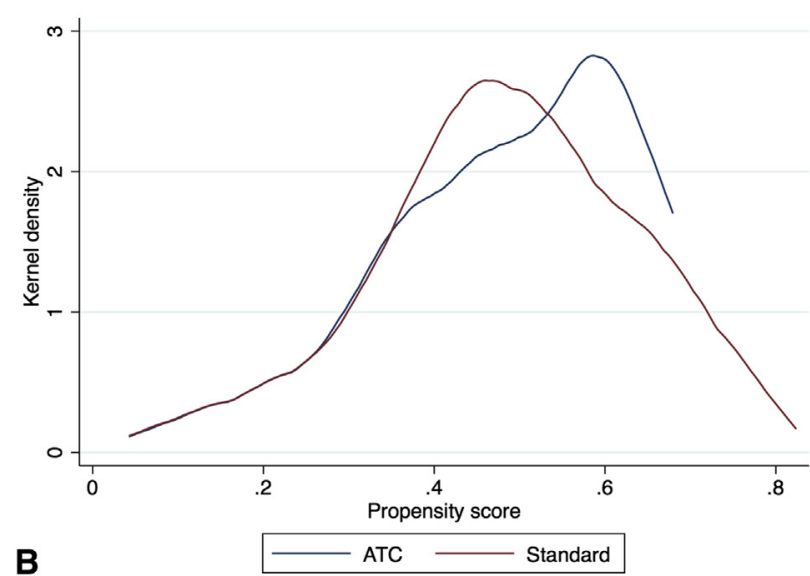

FIGURE 3. Propensity scores kernel density estimation (A) before and (B) after matching. ATC, Active tube clearance.

\section{Outcomes}

Unmatched cohort. Postoperative outcomes are presented in Table 3. Within the general populations, patients managed with ATC chest drainage protocol had a reduction of $34 \%$ in their POAF rate compared with those managed with standard drains $(23 \%$ vs $35 \%, P=.01)$. They required less amiodarone use for AF episode ( $28 \%$ vs $39 \%, P=.03)$ and had shorter hospital length of stay (5.0 [4.0; 7.0] days vs 6.0 [5.0; 8.0] days, $P=.04)$. Matched cohort. In the matched cohort, the ATC group exhibited a trend toward lower incidence of POAF $(24 \%$ vs $35 \%, P=.09$ ) with a relative reduction of $31 \%$. Postoperative hospital length of stay tended to be shorter (5.0 [4.0; $7.0]$ days vs $6.0[5.0 ; 8.0]$ days, $P=.08)$. Total chest tube output (730.0 [500.0; 1250] vs 810.0 [560.0; 1190], $P=.46)$ was similar between ATC and standard drainage groups. There was no difference between groups regarding mortality, cardiac arrest, permanent stroke, transfused allogeneic blood products, or rate of invasive procedure for intrathoracic fluid removal.

\section{Multivariable Analysis}

In the multivariable analysis including the propensity score as a confounder (Table 4), ATC drainage protocol was found to be an independent protective factor for POAF with an odds ratio (OR) of 0.5 (95\% confidence interval $[\mathrm{CI}], 0.1-0.9: P=.02)$. Moreover, age was revealed as a risk factor for POAF with an OR of 1.07 (95\% CI, 1.031.10: $P<.001)$.

\section{DISCUSSION}

POAF is the most common arrhythmia complicating heart surgery, with an incidence ranging from $10 \%$ to $65 \%$ depending on the procedure, patient characteristics, definition, and monitoring. ${ }^{1}$ Development of POAF after heart surgery has been associated with higher risk of hemodynamic compromise, heart failure, and stroke, as well as with longer ICU and hospital stay, greater health care expenditures, and increased mortality. 225-27 Considering these adverse outcomes, prevention of POAF to reduce the aforementioned complications and burdens associated with cardiac surgeries is warranted.

The pathophysiological mechanisms leading to POAF are complex. A trigger able to initiate the tachyarrhythmia and vulnerable atrial substrate capable of sustaining POAF are essential for its generation and can be related to multiple predisposing factors. ${ }^{2}$ Chronic atrial remodeling and fibrosis, such as advancing age and structural heart disease, are preoperative factors increasing the susceptibility to POAF. $^{25,28}$ Intraoperatively, in surgeries involving valvular or aortic procedures, the extensive tissue trauma exacerbates the risk of POAF over coronary artery bypass graft (CABG) alone. ${ }^{1,2}$ In the postoperative setting, transient factors related to surgery predispose to tachyarrhythmias. Sympathetic activation, inflammation, and oxidative stress are all reversible intervention-related mechanisms that account for the generation of POAF after cardiac surgery. ${ }^{25}$ Because they are transitory and alterable, these postoperative factors are the aim for POAF prevention.

Approaches targeting sympathetic activation ( $\beta$-blocker, amiodarone) are actually the only recommended prophylaxis for POAF, but are limited by their adverse effects, such as hypotension and bradycardia. ${ }^{29}$ Meta-analyses have showed corticosteroids and statins can effectively reduce the rate of POAF; however, side effects are deemed too great to justify broad use, and the effect of statins may be limited to specific populations (isolated CABG). ${ }^{4-6}$ Other pharmacological agents aimed at tapering the inflammatory response and oxidative stress (ie, colchicine, vitamins $\mathrm{C}$ and $\mathrm{E}, \mathrm{N}$-acetylcysteine, and omega-3 polyunsaturated fatty acids), have shown promising results in clinical trials but lack satisfactory efficacy to be extensively introduced in clinical practice. ${ }^{7}$ Thus, there is a clear unmet need to find safe and effective prevention strategies for POAF widely applicable without major side effects or specific restriction.

The purpose of this study was to assess whether a modification in drainage protocol aimed at maximizing chest 
TABLE 3. Postoperative outcomes

\begin{tabular}{|c|c|c|c|c|c|c|}
\hline \multirow[b]{2}{*}{ Variable } & \multicolumn{3}{|c|}{ Unadjusted data } & \multicolumn{3}{|c|}{ Propensity score matched data } \\
\hline & $\operatorname{ATC}(n=142)$ & Standard $(n=158)$ & $P$ & $\operatorname{ATC}(n=107)$ & Standard $(n=107)$ & $\boldsymbol{P}$ \\
\hline Atrial fibrillation & $32(23)$ & $56(35)$ & .01 & $26(24)$ & $37(35)$ & .09 \\
\hline $\begin{array}{l}\text { Invasive procedure for intrathoracic } \\
\text { fluid removal (composite) }\end{array}$ & $9(6)$ & $6(4)$ & .31 & $6(6)$ & $4(4)$ & .52 \\
\hline Reexploration & $4(3)$ & $2(1)$ & .43 & $3(3)$ & $1(1)$ & .62 \\
\hline Bleeding & $1(1)$ & $0(0)$ & .48 & $1(1)$ & $0(0)$ & $>.99$ \\
\hline Tamponade & $3(2)$ & $2(1)$ & .67 & $2(2)$ & $1(1)$ & $>.99$ \\
\hline Hemothorax & $1(1)$ & $3(2)$ & .63 & $0(0)$ & $2(2)$ & .50 \\
\hline Pericardial interventions & $2(1)$ & $1(<1)$ & .61 & $2(2)$ & $1(1)$ & $>.99$ \\
\hline Pleural interventions & $4(3)$ & $1(<1)$ & .19 & $3(3)$ & $1(1)$ & .62 \\
\hline Total chest tube output, mL & $730.0(500.0 ; 1235)$ & $855.0(537.5 ; 1215)$ & .41 & $730.0(500.0 ; 1250)$ & $810.0(560.0 ; 1190)$ & .46 \\
\hline Postoperative allogeneic blood products & $53(37)$ & $67(42)$ & .37 & $37(35)$ & $43(40)$ & .40 \\
\hline Erythrocytes & $42(30)$ & $58(37)$ & .19 & $31(29)$ & $36(34)$ & .46 \\
\hline Thrombocytes & $31(22)$ & $30(19)$ & .54 & $19(18)$ & $17(16)$ & .72 \\
\hline Fresh frozen plasma & $10(7)$ & $10(6)$ & .81 & $7(7)$ & $5(5)$ & .55 \\
\hline Amiodarone for AF episode & $39(28)$ & $61(39)$ & .03 & $31(29)$ & $37(35)$ & .33 \\
\hline Warfarin for recurrent $\mathrm{AF}$ & $7(5)$ & $7(5)$ & .87 & $6(6)$ & $5(5)$ & .78 \\
\hline Stroke & $2(1)$ & $1(<1)$ & .61 & $2(2)$ & $1(1)$ & $>.99$ \\
\hline Cardiac arrest & $1(<1)$ & $0(0)$ & .47 & $1(1)$ & $0(0)$ & $>.99$ \\
\hline Acute kidney injury & $16(11)$ & $10(6)$ & .15 & $13(12)$ & $7(7)$ & .19 \\
\hline Sternal infection & $1(<1)$ & $4(3)$ & .37 & $0(0)$ & $3(3)$ & .25 \\
\hline Total ventilation time, $\mathrm{h}$ & $4.83(3.48 ; 8.13)$ & $5.00(4.15 ; 6.25)$ & .73 & $5.29(3.77 ; 9.17)$ & $5.20(4.33 ; 7.19)$ & .66 \\
\hline Total ICU time, $\mathrm{h}$ & $27.8(23.3 ; 50.6)$ & $26.9(22.3 ; 70.7)$ & .74 & $27.8(23.0 ; 51.0)$ & $27.0(22.3 ; 70.5)$ & .88 \\
\hline Total hospital length of stay, $d$ & $5.0(4.0 ; 7.0)$ & $6.0(5.0 ; 8.0)$ & .04 & $5.0(4.0 ; 7.0)$ & $6.0(5.0 ; 8.0)$ & .08 \\
\hline Mortality & $5(4)$ & $4(3)$ & .74 & $5(5)$ & $1(1)$ & .21 \\
\hline
\end{tabular}

Categorical variables are presented as frequency (\%); continuous variables are presented as median (IQR). $A T C$, Active tube clearance; $A F$, atrial fibrillation; $I C U$, intensive care unit.

tube patency could effectively and safely prevent POAF, thus providing the same benefits as posterior pericardiotomy, but without the risk of vein graft compression and bleeding from the additional pericardial incision, as well as long-term adherences and cardiac herniation. ${ }^{15,30}$ Our investigation did, in fact, when comparing the 300 patients of the general population, demonstrate a statistically significant reduction in the rate of POAF of $34 \%$ in favor of chest drainage using ATC (23\% vs $35 \%$ ). The propensity-matched cohort, analyzing 214 patients, exhibited similar incidence rates of POAF $(24 \%$ vs

TABLE 4. Predictors of postoperative atrial fibrillation on multivariable analysis in the unmatched cohort

\begin{tabular}{lcccc}
\hline \multicolumn{1}{c}{ Variable } & Coefficient $(\boldsymbol{\beta})$ & Odds ratio & $\mathbf{9 5} \% \mathbf{C I}$ & $\boldsymbol{P}$ \\
\hline Active tube clearance & -2.3 & 0.5 & $0.1-0.9$ & .02 \\
Age, y & 3.7 & 1.07 & $1.03-1.10$ & $<.001$ \\
Arterial hypertension & 1.1 & 1.8 & $0.6-4.9$ & .27 \\
NYHA $\geq$ III & 1.7 & 2.5 & $0.8-7.5$ & .10 \\
Preoperative CCB & 0.9 & 1.4 & $0.7-2.7$ & .36 \\
Valve replacement & 3.7 & 1.2 & $0.4-4.2$ & .75 \\
Ascending aortic & 0.5 & 1.4 & $0.4-4.9$ & .60 \\
$\quad$ procedure & & & & \\
Propensity score & 0.6 & 1.8 & $0.3-10.3$ & .53 \\
Intercept & -4.7 & - & - & - \\
\hline Likelihood ratio $\chi^{2}$ of 42 with a $P$ value $<.001$ and a C-statistic of $0.7 . C I$, Confidence \\
interval; $N Y H A$, New York Heart Association; $C C B$, calcium channel blocker.
\end{tabular}

$35 \%$ ) representing a reduction of $31 \%$ with ATC, while not reaching statistical significance. The multivariable analysis integrating the propensity score as covariate allowing for maximized statistical power and lessened loss of data, however, demonstrated a significant effect related to ATC with the odds of an event of POAF being $50 \%$ less in the ATC group than in the standard drainage group. These results correlate with the $33 \%$ reduction associated with active clearance of chest tubes revealed in a recent propensity-matched investigation by Sirch et al. ${ }^{17}$

The mechanism by which ATC drainage reduces POAF remains to be defined. The original assumption rested on the rationale that more patent catheters would reduce blood pooling within the pericardial space by extracting more fluid, thus reducing cardiomyocyte damage from inflammation and oxidative stress. ${ }^{11,31,32}$ However, our measures of drainage efficiency (ie, total chest tube output, transfusion requirement, and invasive procedure for intrathoracic fluid removal) remained similar between ATC and standard drainage. Furthermore, in Sirch et al., ${ }^{17}$ ATC was even associated with a smaller quantity of drained blood. This does not rule out the possibility that the composition of the pericardial blood rather than its quantity could be the predominant factor driving POAF generation in susceptible individuals. 


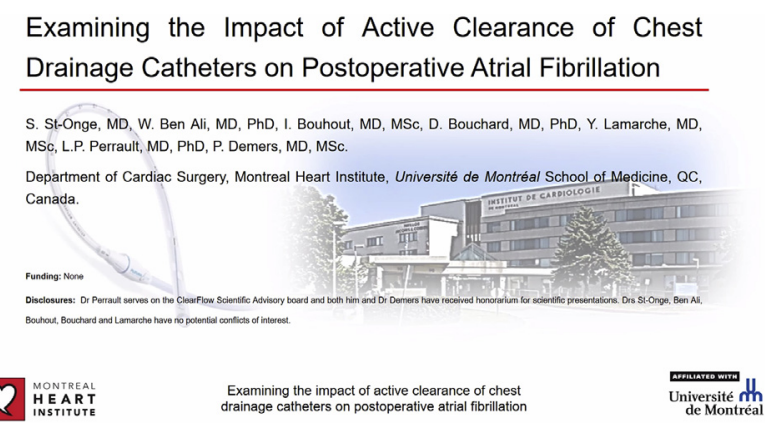

VIDEO 1. Active clearance of chest drainage catheters, an encouraging method to prevent postoperative atrial fibrillation at bedside. Video available at: http://www.jtcvsonline.org/article/S0022-5223(17)30556-1/addons.

In fact, we hypothesize that for the same amount of drained blood, an unobstructed catheter could allow steadier drainage giving less opportunity for blood to pool around the heart and undergo pro-oxidative modification inside of the thoracic cavity. ${ }^{12,13}$ Such alterations in the composition of pericardial fluid after cardiac surgery (ie, high concentration of oxidized hemoglobin, lipid peroxidation products, cardiac injury markers, and inflammatory cell migration) have been demonstrated Kramer et al. ${ }^{11}$ According to their work, pooling blood could result in erythrocyte hemolysis, releasing cell-free hemoglobin within the pericardium, which would induce the migration of neutrophils and subsequent release of reactive oxygen species (ROS) as part of the oxidative burst. Inadequately drained blood could also, by activation of the coagulation cascade and generation of thrombin and fibrin, which have strong chemotactic properties, crosstalk with inflammatory response mediators, thus promoting migration of neutrophils and ensuing oxidative stress. ${ }^{10,16,33,34}$ Through both mechanisms, the lipid peroxidation caused by ROS would then result in the breakdown of cardiomyocyte cell membrane and electromechanical disturbances of the atrial tissue, rendering it more prompt to trigger arrhythmia after cardiac surgery. Further investigations are necessary to demonstrate whether or not ATC, by keeping clear and functional catheters, is able to exert better control on local pericardial inflammation and reduce cardiomyocyte exposition to oxidative stress caused by pooling blood, explaining the reduced incidence of POAF. ${ }^{3}$

This study provides more insight on a new potential device that could be used to prevent the triggering of arrhythmia after heart surgery and improve patients' recovery. This is of particular interest in light of the new study by Gillinov and colleagues ${ }^{18}$ showing no net advantage between rate control and rhythm control for the management of POAF and the tapering of its burden, therefore increasing the importance of adequate upstream prevention. Moreover, the trend toward reduction in hospital length of stay is of particular economic interest and, if confirmed by other investigations, might help to justify the cost of this technology.

\section{Limitations}

This retrospective study of prospectively collected data has several limitations. The sample size of patients who were allocated to ATC drainage was small, and after matching was reduced by more than $25 \%$, which likely impaired statistical power regarding the matched cohort. Also, despite the matching process and multivariable analysis, there probably remain hidden differences between the 2 groups, perhaps related to observer bias and nonsynchronous control, which only the prospective randomized trial currently being carried out at our institution could overcome. The management of standard chest tubes (ie, numbers and locations) was not protocolized and could have partly accounted for the observed difference in the rate of POAF between groups. The lack of consistent preoperative pharmacologic prophylaxis in either group could be a cause of concern and needs to be recognized. Moreover, in the retrospective setting, it has been difficult to assess the exact duration of POAF episodes. The fact that the definition for POAF is variable in the literature must be acknowledged when comparing studies. Finally, some data elements that could have given more insight about the pathophysiological effect of ATC were not collected in this study, especially retained pericardial blood directly measured by imaging techniques and analysis of inflammatory and oxidative injury markers within the drainage fluid. These are important considerations for further investigations.

\section{CONCLUSIONS}

Implementation of an ATC chest drainage protocol may be associated with a reduction of POAF. Our findings highlight the importance of maintaining chest tube patency after cardiac surgery and unveil the use of ATC as a potential method of chest tube management to prevent POAF (Video 1). Because all patients need chest tubes after heart surgery, this shows promise as a potentially safe and efficacious preventive measure that can be widely applied without the side effects that limit the use of $\beta$-blockers and other pharmacological approaches or the risks associated with posterior pericardiotomy. A randomized controlled trial is needed to confirm our findings. Further studies will be useful to evaluate the extent of the benefits provided by this system and demonstrate explicitly the physiological effect by which active clearance of drainage catheters might confer protection against POAF.

\section{Conflict of Interest Statement}

Dr Perrault serves on the ClearFlow Scientific Advisory board and both Dr Perrault and Dr Demers have received 
honoraria for scientific presentations. The investigators had full unrestricted scientific control of the data that were independently analyzed. All other authors have nothing to disclose with regard to commercial support.

\section{References}

1. Maisel WH, Rawn JD, Stevenson WG. Atrial fibrillation after cardiac surgery. Ann Intern Med. 2001;135:1061-73.

2. LaPar DJ, Speir AM, Crosby IK, Fonner E Jr, Brown M, Rich JB, et al. Postoperative atrial fibrillation significantly increases mortality, hospital readmission, and hospital costs. Ann Thorac Surg. 2014;98:527-33.

3. Biancari F, Mahar MA. Meta-analysis of randomized trials on the efficacy of posterior pericardiotomy in preventing atrial fibrillation after coronary artery bypass surgery. J Thorac Cardiovasc Surg. 2010;139:1158-61.

4. Ho KM, Tan JA. Benefits and risks of corticosteroid prophylaxis in adult cardiac surgery: a dose-response meta-analysis. Circulation. 2009;119: 1853-66.

5. Zheng H, Xue S, Hu ZL, Shan JG, Yang WG. The use of statins to prevent postoperative atrial fibrillation after coronary artery bypass grafting: a meta-analysis of 12 studies. J Cardiovasc Pharmacol. 2014;64:285-92.

6. Bolesta S, Kong F. Effect of statins on the incidence of postoperative atrial fibrillation after cardiac valve surgery. Pharmacotherapy. 2015;35:998-1006.

7. Zakkar M, Ascione R, James AF, Angelini GD, Suleiman MS. Inflammation, oxidative stress and postoperative atrial fibrillation in cardiac surgery. Pharmacol Ther. 2015;154:13-20.

8. Davis EM, Packard KA, Hilleman DE. Pharmacologic prophylaxis of postoperative atrial fibrillation in patients undergoing cardiac surgery: beyond betablockers. Pharmacotherapy. 2010;30:274e-318e.

9. Tokmaji G, McClure RS, Kaneko T, Aranki SF. Management strategies in cardiac surgery for postoperative atrial fibrillation: contemporary prophylaxis and futuristic anticoagulant possibilities. Cardiol Res Pract. 2013;2013:637482.

10. Boyle EM Jr, Gillinov AM, Cohn WE, Ley SJ, Fischlein T, Perrault LP. Retained blood syndrome after cardiac surgery: a new look at an old problem. Innovations (Phila). 2015;10:296-303.

11. Kramer PA, Chacko BK, Ravi S, Johnson MS, Mitchell T, Barnes S, et al. Hemoglobin-associated oxidative stress in the pericardial compartment of postoperative cardiac surgery patients. Lab Invest. 2015;95:132-41.

12. Wu JH, Marchioli R, Silletta MG, Masson S, Sellke FW, Libby P, et al. Oxidative Stress Biomarkers and Incidence of Postoperative Atrial Fibrillation in the Omega-3 Fatty Acids for Prevention of Postoperative Atrial Fibrillation (OPERA) Trial. J Am Heart Assoc. 2015;4:e001886.

13. Ramlawi B, Otu H, Mieno S, Boodhwani M, Sodha NR, Clements RT, et al. Oxidative stress and atrial fibrillation after cardiac surgery: a case-control study. Ann Thorac Surg. 2007;84:1166-72.

14. Hu XL, Chen Y, Zhou ZD, Ying J, Hu YH, Xu GH. Posterior pericardiotomy for the prevention of atrial fibrillation after coronary artery bypass grafting: a metaanalysis of randomized controlled trials. Int J Cardiol. 2016;215:252-6.

15. Gozdek M, Pawliszak W, Hagner W, Zalewski P, Kowalewski J, Paparella D, et al. Systematic review and meta-analysis of randomized controlled trials assessing safety and efficacy of posterior pericardial drainage in patients undergoing heart surgery. J Thorac Cardiovasc Surg. 2017;153:865-75.e12.

16. Karimov JH, Gillinov AM, Schenck L, Cook M, Kosty Sweeney D, Boyle EM, et al. Incidence of chest tube clogging after cardiac surgery: a single-centre prospective observational study. Eur J Cardiothorac Surg. 2013;44:1029-36.
17. Sirch J, Ledwon M, Puski T, Boyle EM Jr, Pfeiffer S, Fischlein T. Active clearance of chest drainage catheters reduces retained blood. J Thorac Cardiovasc Surg. 2016;151:832-8.e2.

18. Gillinov AM, Bagiella E, Moskowitz AJ, Raiten JM, Groh MA, Bowdish ME, et al. Rate control versus rhythm control for atrial fibrillation after cardiac surgery. N Engl J Med. 2016;374:1911-21.

19. Balzer F, von Heymann C, Boyle EM, Wernecke KD, Grubitzsch H, Sander M Impact of retained blood requiring reintervention on outcomes after cardiac surgery. J Thorac Cardiovasc Surg. 2016;152:595-601.e4.

20. Perrault LP, Pellerin M, Carrier M, Cartier R, Bouchard D, Demers P, et al. The PleuraFlow Active Chest Tube Clearance System: initial clinical experience in adult cardiac surgery. Innovations (Phila). 2012;7:354-8.

21. Shalli S, Boyle EM, Saeed D, Fukamachi K, Cohn WE, Gillinov AM. The active tube clearance system: a novel bedside chest-tube clearance device. Innovations (Phila). 2010;5:42-7.

22. Day TG, Perring RR, Gofton K. Is manipulation of mediastinal chest drains use ful or harmful after cardiac surgery? Interact Cardiovasc Thorac Surg. 2008; 888-90.

23. Mitchell LB. Canadian Cardiovascular Society atrial fibrillation guidelines 2010: prevention and treatment of atrial fibrillation following cardiac surgery. Can J Cardiol. 2011:27:91-7.

24. January CT, Wann LS, Alpert JS, Calkins H, Cigarroa JE, Cleveland JJC, et al. 2014 AHA/ACC/HRS guideline for the management of patients with atrial fibrillation: executive summary-a report of the American College of Cardiology/ American Heart Association Task Force on Practice Guidelines and the Heart Rhythm Society. J Am Coll Cardiol. 2014;64:2246-80.

25. Maesen B, Nijs J, Maessen J, Allessie M, Schotten U. Post-operative atrial fibrillation: a maze of mechanisms. Europace. 2012;14:159-74.

26. Lahtinen J, Biancari F, Salmela E, Mosorin M, Satta J, Rainio P, et al. Postoperative atrial fibrillation is a major cause of stroke after on-pump coronary artery bypass surgery. Ann Thorac Surg. 2004;77:1241-4.

27. Burgess DC, Kilborn MJ, Keech AC. Interventions for prevention of postoperative atrial fibrillation and its complications after cardiac surgery: a metaanalysis. Eur Heart J. 2006;27:2846-57.

28. Frendl G, Sodickson AC, Chung MK, Waldo AL, Gersh BJ, Tisdale JE, et al 2014 AATS guidelines for the prevention and management of perioperative atrial fibrillation and flutter for thoracic surgical procedures. J Thorac Cardiovasc Surg. 2014;148:e153-93.

29. Baker WL, White CM. Post-cardiothoracic surgery atrial fibrillation: a review of preventive strategies. Ann Pharmacother. 2007;41:587-98.

30. Conti VR. Draining the posterior pericardial space; pericardiotomy vs. just another tube. J Thorac Cardiovasc Surg. 2017;153:876-7.

31. Shiose A, Takaseya T, Fumoto H, Arakawa Y, Horai T, Boyle EM, et al. Improved drainage with active chest tube clearance. Interact Cardiovasc Thorac Surg. 2010;10:685-8.

32. Arakawa Y, Shiose A, Takaseya T, Fumoto H, Kim HI, Boyle EM, et al. Superior chest drainage with an active tube clearance system: evaluation of a downsized chest tube. Ann Thorac Surg. 2011;91:580-3.

33. Levi M, van der Poll T, Buller HR. Bidirectional relation between inflammation and coagulation. Circulation. 2004;109:2698-704.

34. Chen D, Dorling A. Critical roles for thrombin in acute and chronic inflammation. J Thromb Haemost. 2009;7:122-6.

Key Words: atrial fibrillation, cardiac surgery, pericardial drainage, perioperative care 